\title{
Optical Conductivity of Two-Dimensional Silicon: Evidence of Dirac Electrodynamics
}

This document is the Accepted Manuscript version of a Published Work that appeared in final form in Nano Letters, copyright (C) American Chemical Society after peer review and technical editing by the publisher. To access the final edited and published work see https://pubs.acs.org/doi/10.1021/acs.nanolett.8b03169.

Carlo Grazianetti ${ }^{1}$, Stefania De Rosa ${ }^{2}$, Christian Martella $^{1}$, Paolo Targa $^{3}$, Davide Codegoni ${ }^{3}$, Paola Gori $^{4}$, Olivia Pulci ${ }^{5,6}$, Alessandro Molle ${ }^{1, *}$, Stefano Lupi ${ }^{2, *}$

${ }^{1}$ CNR-IMM Unit of Agrate Brianza, via C. Olivetti 2, Agrate Brianza, I-20864, Italy

${ }^{2}$ CNR-IOM Dipartimento di Fisica, Università di Roma La Sapienza, p.le Aldo Moro 2, Roma, I-00185, Italy

${ }^{3}$ STMicroelectronics, via C. Olivetti 2, Agrate Brianza, I-20864, Italy

${ }^{4}$ Dipartimento di Ingegneria, Università Roma Tre, via della Vasca Navale 79, I-00146, Roma, Italy

${ }^{5}$ Dipartimento di Fisica, Università di Roma Tor Vergata, via della Ricerca Scientifica 1, Roma, I-00133, Italy

${ }^{6}$ INFN, Sezione di Roma Tor Vergata, via della Ricerca Scientifica 1, Roma, I-00133, Italy

*Corresponding Authors: Prof. Stefano Lupi (stefano.lupi@roma1.infn.it) and Dr. Alessandro Molle (alessandro.molle@mdm.imm.cnr.it)

Keywords: two-dimensional; silicon; silicene; $\mathrm{Al}_{2} \mathrm{O}_{3}(0001)$; optical conductivity; DFT calculations.

\begin{abstract}
The exotic electrodynamics properties of graphene come from the linearly dispersive electronic bands that host massless Dirac electrons. A similar behavior was predicted to manifest in freestanding silicene, the silicon counterpart of graphene, thereby envisaging a new route for a silicon photonics. However, the access to silicene exploitation in photonics was hindered so far by the use of optically inappropriate substrates in experimentally realized silicene. Here we report on the optical conductivity of silicon nanosheets epitaxially grown on the optically transparent $\mathrm{Al}_{2} \mathrm{O}_{3}(0001)$ from a thickness of a few tens of nanometers down to the extreme twodimensional (2D) limit. When approaching a 2D regime, a Dirac-like electrodynamics can be deduced from the observation of a low-energy optical conductivity feature owing to a silicene-based interfacing to the substrate.
\end{abstract}


Since its rise, graphene has been fostering unprecedented advances in a number of multidisciplinary applications. ${ }^{1}$ A ubiquitous exploitation of graphene is limited by integration issues in many nanotechnology branches that are still based on silicon. Nowadays, silicon still offers the unique potential to co-integrate electronics and photonics at the nanoscale on a single chip. ${ }^{2}$ Reducing silicon to a graphene-like form would bring to a substantial technology throughput in this framework. The recently discovered two-dimensional (2D) allotropic phase of silicon, namely silicene, followed up by other X-enes (X belongs to group-IIIA, -IVA, and -VA) renewed the interest in silicon-based nanomaterials as candidates for applications in nanotechnology. ${ }^{3,4}$ In fact, dimensional reduction of silicon opens new and intriguing routes for silicon nanoelectronics and photonics, like engineered and tunable in-gap absorption for photovoltaic application. Hitherto, most of the published reports are related to silicene growth on metallic templates, e.g. $\operatorname{Ag}(111)$, hence these substrates cannot be easily used to directly access the optical properties of silicene and, more generally, of most of the $\mathrm{X}$-enes sharing the same issue. ${ }^{5}$ Nonetheless, even the optical properties of silicene, as well as the electronic ones, are predicted to closely resemble those of the forerunning graphene. In particular, ideal freestanding silicene shares with graphene the low-frequency electrodynamics, characterized by a universal absorption value $\pi \alpha$ where $\alpha$ is the fine-structure constant. ${ }^{6-8}$ Intriguingly, the rise of massless Dirac fermions at lowenergy occurs despite either the buckling amount or the mixed $s p^{2}-s p^{3}$ hybridization. ${ }^{6}$ For higher-energy, the theoretical absorbance spectrum of freestanding silicene is characterized by two main interband transitions at $\mathrm{M}\left(\pi \rightarrow \pi^{*}\right.$ transition, hereafter termed I) and $\Gamma\left(\sigma \rightarrow \sigma^{*}\right.$ transition, termed II) points of the first Brillouin zone (BZ) at 1.6 and $4 \mathrm{eV}$, respectively, corresponding to van Hove singularities of the joint density of states (JDOS) ${ }^{6-8}$ Nonetheless, when silicene is supported by a metallic substrate, most of these properties vanishes as far as spurious hybridization come into play. For instance, for the silicene on $\mathrm{Ag}(111)$ case, the strong SiAg hybridization has been proven to deeply affect the electronic and optical properties of the supported silicene, ${ }^{9,10}$ giving rise to a complicated absorbance spectrum showing superimposition of different contributions with mixed Si-Ag and Ag-Ag transitions. ${ }^{11}$ In this light, many efforts should be then devoted to the synthesis of silicene or, generally speaking, of low-dimensional silicon nanosheets (SiNSs), on dielectric (transparent) substrates in order to reduce the interaction, providing a more ideal case to investigate silicene 
optical properties and therein enabling a silicene-based photonics. In this framework, $\mathrm{Al}_{2} \mathrm{O}_{3}(0001)$, with a reported experimental bandgap of $8.8 \mathrm{eV},{ }^{12}$ has been recently proposed as a commensurate substrate either for silicene or for germanene epitaxy. ${ }^{13}$ Indeed, based on first-principles calculations, Chen et al. suggested that the Al-terminated surface of $\mathrm{Al}_{2} \mathrm{O}_{3}(0001)$ can stabilize a monolayer honeycomb structure of silicene (also germanene) without destroying the Dirac states, because the substrate, being a large-gap semiconductor with a proper work function, imposes the Dirac point to lie in the gap and far from the substrate states when their bands align. Silicene on $\mathrm{Al}_{2} \mathrm{O}_{3}(0001)$ retains the main structural profile of the low-buckled honeycomb lattice. ${ }^{13}$ Stimulated by this theoretical prediction, here we report on the innovative synthesis of SiNSs on $\mathrm{Al}_{2} \mathrm{O}_{3}(0001)$ substrates by molecular beam epitaxy (MBE), under carefully tailored conditions, and on the related optical response from infrared (IR) to ultraviolet (UV). The first time measured optical conductivity shows a low-energy electrodynamics behavior in agreement with a Dirac-like electronic dispersion. This experimental result is further corroborated and critically interpreted by density functional theory (DFT) $a b$ initio calculations of the structural, electronic, and optical properties based on a silicene model. Our outcomes demonstrate that, at the pure 2D limit, SiNSs grown on $\mathrm{Al}_{2} \mathrm{O}_{3}(0001)$ retain the properties of freestanding silicene in their optical conductivity.

In order to get through the optical properties of silicon at the 2D limit, three main issues should be firstly addressed, i.e. silicon growth, precise thickness determination, integrity and stability of specifically designed samples. Hence, we studied samples with different SiNSs thicknesses down to $0.5 \mathrm{~nm}$ (see Methods) on the ultra-high vacuum (UHV) prepared $\mathrm{Al}_{2} \mathrm{O}_{3}(0001)$ surface, which is Al-terminated. ${ }^{14,15}$ However, to take into account substrate-induced effects on the optical measurements, we fabricated a specific type of sample with variable silicon thickness, which ranges from 1.5 to $7 \mathrm{~nm}$ through $1 \mathrm{~cm}$ wide $\mathrm{Al}_{2} \mathrm{O}_{3}$ substrate, as well as a constant thickness $(\mathrm{CT})(0.5 \mathrm{~nm})$ sample at the pure 2D limit (Figure 1a). 
a

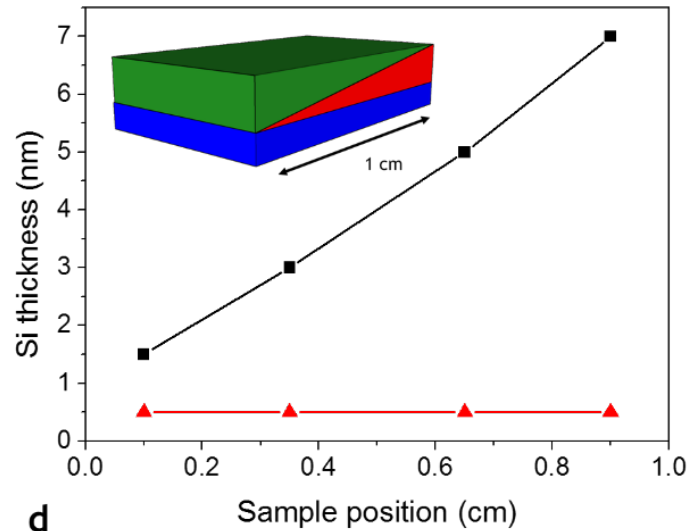

d

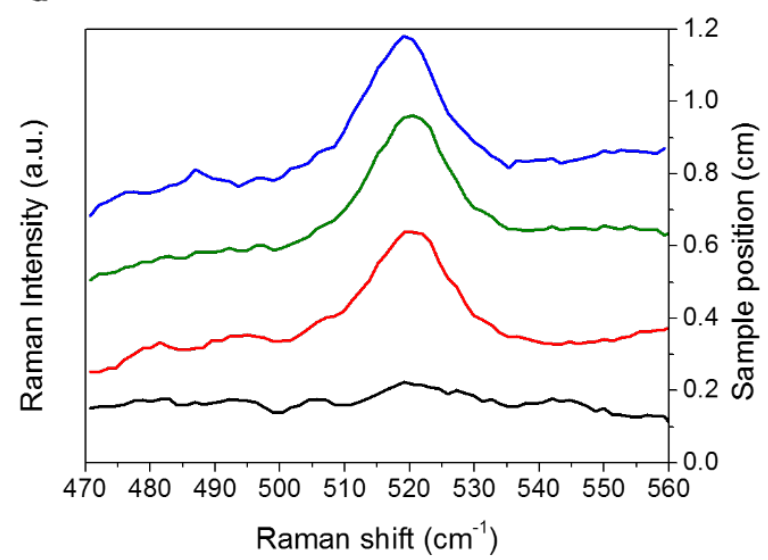

b
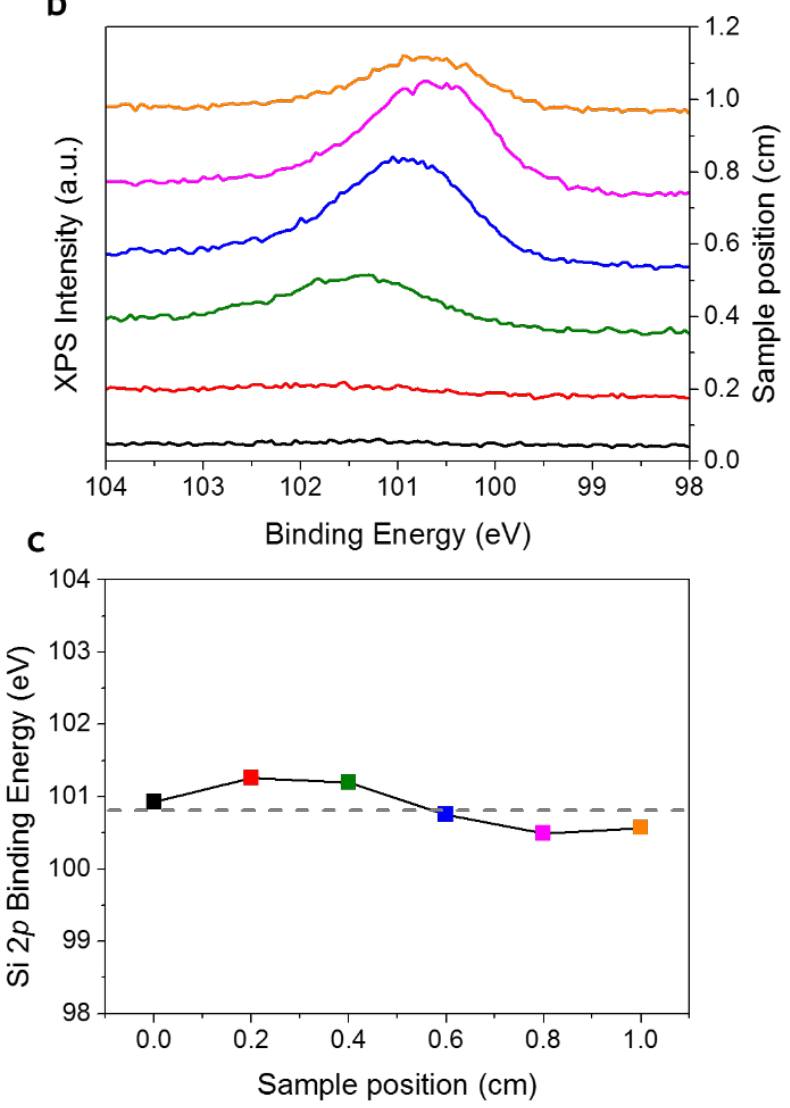

Figure 1. (a) SiNSs thickness by AFM for the VT sample (black dots, line is a guide for the eye) and the CT sample (red triangles). Inset displays schematics of the $\mathrm{Al}_{2} \mathrm{O}_{3}$-capped (green) VT sample showing the increasing silicon thickness (red) along one $\mathrm{Al}_{2} \mathrm{O}_{3}(0001)$ substrate (blue) direction. (b) XPS spectra and (c) binding energy of the Si $2 p$ core level along the increasing silicon thickness of the VT sample, where grey dashed line is the average value (black line is a guide for the eye). (d) Raman spectroscopy of the first-order mode along the increasing thickness direction as for (b).

The former one is deemed to illustrate the optical behavior of the increasing silicon thickness of which the latter is the lower limit. A $25 \mathrm{~nm}$-thick amorphous sample was further grown at room temperature with the same MBE technique, as reference for comparison. Precise control on the SiNSs thickness is confirmed through ex situ atomic force microscopy (AFM) survey (see Figure 1a and Supporting Information Figures S1-S3) and plays a crucial role for the optical properties. The variable thickness (VT) SiNS sample allowed us to access the properties of the SiNSs avoiding background issues related to substrate variability and it is schematically depicted in the inset of Figure 1a. In situ x-ray photoelectron spectroscopy (XPS) was carried out along the slope of the VT sample to check the chemical status of the SiNSs after the growth. Figure 1b 
shows that $\mathrm{Si} 2 p$ core level is placed on average at $100.89 \mathrm{eV}$ thus resulting in a moderate shift to higher binding energy than that of silicene on $\operatorname{Ag}(111),{ }^{16}$ on $\operatorname{MoS}_{2}(0002),{ }^{17}$ and on $\mathrm{ZrB}_{2}(0001) .{ }^{18}$ Figure 1c indicates there is a small variation in binding energy along the thickness slope (within the experimental error of \pm 0.08 $\mathrm{eV}$ ), with a higher binding energy for the thinner part of the VT sample, i.e. for the SiNSs in close contact with the substrate. Furthermore, the observed shift of the silicon core level to higher binding energy is strictly related to the low-dimensionality of the SiNSs, as demonstrated by its thickness-dependent behavior (Figure 1c), which refers to the change in the (size-dependent) ionization potential. ${ }^{19}$ Indeed, a similar binding energy $(100.73 \mathrm{eV})$ is found even in the thinnest CT investigated sample (see Supporting Information Figure S2). On the other hand, the binding energy of bulk silicon $(\sim 99 \mathrm{eV})$ is almost recovered for the $\sim 25 \mathrm{~nm}$-thick amorphous sample (see Supporting Information Figure S3), in good agreement with the optical measurements (see below). In order to prevent the oxidation when taking samples out of UHV environment, SiNSs were encapsulated with $5 \mathrm{~nm}$-thick $\mathrm{Al}_{2} \mathrm{O}_{3}$ amorphous capping layer (see Methods and Ref. ${ }^{16}$ ). After capping, the Si $2 p$ core level shifts to lower binding energy $(99.54 \mathrm{eV})$. This shift is likely related to charge transfer between silicon and amorphous oxide at the interface, as reported for silicene on $\operatorname{Ag}(111),{ }^{16}$ rather than chemical modifications within the SiNSs. Indeed, no signatures of silicon oxides have been disclosed throughout the XPS analysis and also confirmed by the unchanged full width half maximum values of the $\mathrm{Al} 2 p$ and $\mathrm{O} 1 s$ core levels before and after silicon deposition (Supporting Information Table S1). Moreover, this amorphous capping layer does not affect the optical properties of SiNSs on $\mathrm{Al}_{2} \mathrm{O}_{3}(0001)$, due to its small thickness and intrinsic transparency. Hence, top and bottom interfaces of SiNSs are protected by transparent films. Accordingly, the Raman scattering investigation depicted in Figure 1d shows the first-order Raman mode of the encapsulated SiNSs at different spatial positions along the thickness slope of the VT sample. At each position, a clear Raman feature is observed at $\sim 520.5 \mathrm{~cm}^{-1}$, close to the $F_{2 \mathrm{~g}}$ mode of cubic silicon at $520.6 \mathrm{~cm}^{-1}$ (or equivalently of thin siliconon-insulator films), ${ }^{20}$ thus confirming the presence of the SiNSs even after the encapsulation process and the subsequent exposure of the samples to ambient condition. Furthermore, the increase of the Raman mode intensity as a function of the spatial position (along the direction shown in the inset of Figure 1a) confirms the thickness variation along the slope in agreement with the AFM and XPS measurements. The low-intensity of the Raman mode in the thinnest part of the VT sample (black spectrum in Figure 1d) can be likely related to the small Raman scattering efficiency for the encapsulated SiNSs at the used laser frequency. This similarly 
occurs on the CT sample. These Raman modes were successfully recovered even months after the growth, without hints of amorphous or deteriorated silicon, thus confirming the stability of the encapsulated SiNSs in ambient conditions. Combining XPS and Raman spectroscopy, the effectiveness of the $\mathrm{Al}_{2} \mathrm{O}_{3}$ capping layer to durably protect 2D silicon on substrates other than $\mathrm{Ag}(111)$ is demonstrated. It is not surprising to find out a Raman mode placed so close to those of bulk $\mathrm{Si}(111)$ and silicene on $\operatorname{Ag}(111)$, as far as the predicted $\mathrm{Si}-\mathrm{Si}$ bond length $l$ in silicene on $\mathrm{Al}_{2} \mathrm{O}_{3}(0001)(2.34<l<2.37 \AA$, see also Supplementary Information) is very close to those of bulk $\operatorname{Si}(111)(2.34 \AA)$ or silicene on $\operatorname{Ag}(111)(2.28<l<2.39 \AA) .{ }^{21}$ Additional evidence on the SiNSs growth is provided by transmission electron microscopy (TEM) imaging that shows the formation of a continuous crystalline silicon film sandwiched in between the amorphous $\mathrm{Al}_{2} \mathrm{O}_{3}$ capping layer and the $\mathrm{Al}_{2} \mathrm{O}_{3}(0001)$ substrate (Figure 2a).
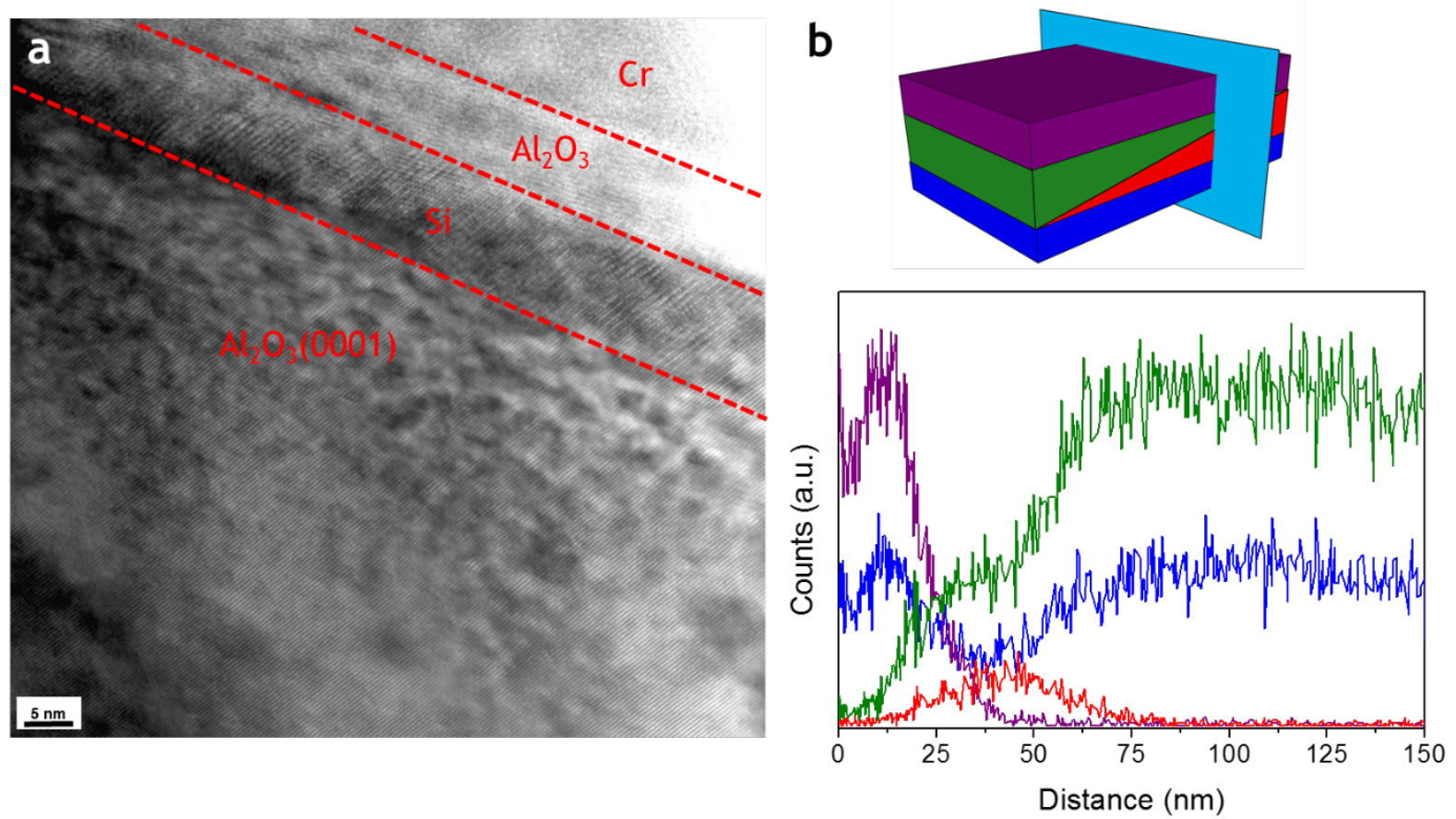

Figure 2. (a) TEM image of the cross-section view (from top to bottom) of the $\mathrm{Cr} / \mathrm{a}-\mathrm{Al}_{2} \mathrm{O}_{3} / \mathrm{Si} / \mathrm{Al}_{2} \mathrm{O}_{3}(0001$ ) stack. (b) Sketch of the stacking cross sectional cut (top) and EDX line scans of $\mathrm{K}_{\alpha 1}$ of chromium (purple), aluminum (green), silicon (red), and oxygen (blue) (bottom) extracted from the respective maps reported in Figure S4.

Higher-resolution TEM images are hampered by the well-known issue on the electron beam-induced crystallization of the amorphous $\mathrm{Al}_{2} \mathrm{O}_{3}$, ${ }^{22}$ with a progressive deterioration of the whole lamella in a few seconds. However, the SiNSs in the thicker part of VT sample (see cross-section cut in Figure 2b) are clearly 
confined without any chemical intermixing between the substrate and the capping layer, as evidenced by the energy dispersive x-rays (EDX) analysis (Figure 2b), thus suggesting a chemically protected environment for the SiNSs. Line profiles of the corresponding EDX maps (reported in Supporting Information Figure S4) shows that the silicon signal (red curve) is maximum where both aluminum (green curve) and oxygen (blue curve) have a (local) minimum intensity.

The absolute optical transmittance T( $\omega)$ (Supporting Information) was measured on CT $(0.5 \mathrm{~nm})$, VT (from 1.5 to $7 \mathrm{~nm}), 25 \mathrm{~nm}$ "bulk" samples, and on the bare $\mathrm{Al}_{2} \mathrm{O}_{3}(0001)$ substrate, in the photon frequency $(\omega)$ range from IR $(0.25 \mathrm{eV})$ to $\mathrm{UV}(4.5 \mathrm{eV})$. It is worth noting that outside this energy range and in particular in the midIR range below $0.25 \mathrm{eV}, \mathrm{Al}_{2} \mathrm{O}_{3}(0001)$ strongly absorbs, thereby preventing a reliable transmittance measurement of SiNSs films. From the knowledge of the real and the imaginary parts of the refraction index of $\mathrm{Al}_{2} \mathrm{O}_{3}(0001)$ substrate (determined from its absolute transmittance and shown in Supporting Information Figure S5) and of T( $\omega)$ of SiNSs we have determined the optical conductivity $\sigma(\omega)=\sigma_{1}(\omega)+i \sigma_{2}(\omega)$ of SiNSs through the use of a Kramers-Kronig constrained fit (see Methods and Supporting Information). Figure 3a shows the real part of the optical conductivity for different thicknesses. $\sigma_{1}(\omega)$ at the lowest thickness (CT sample, $0.5 \mathrm{~nm}$, black curve in Figure 3a) shows a small absorption peak around $1.4 \mathrm{eV}$ superimposed to a nearly flat background in the whole IR range. 


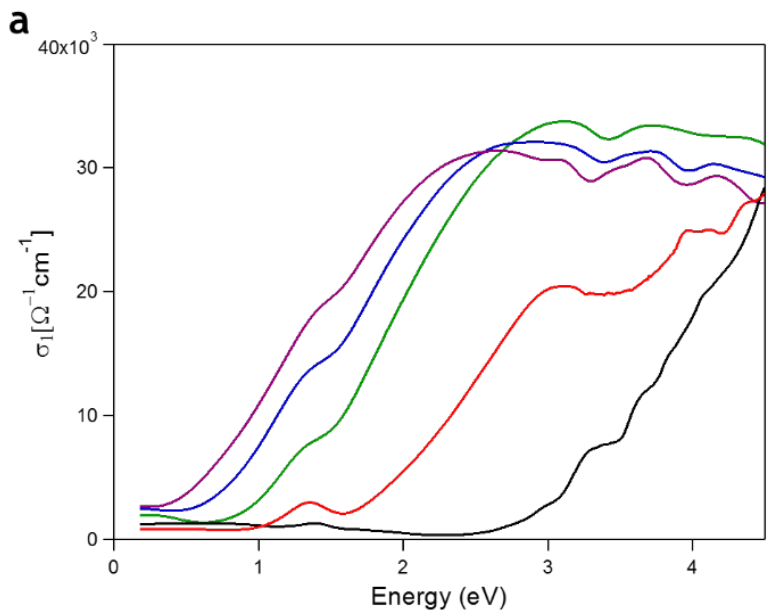

b

C
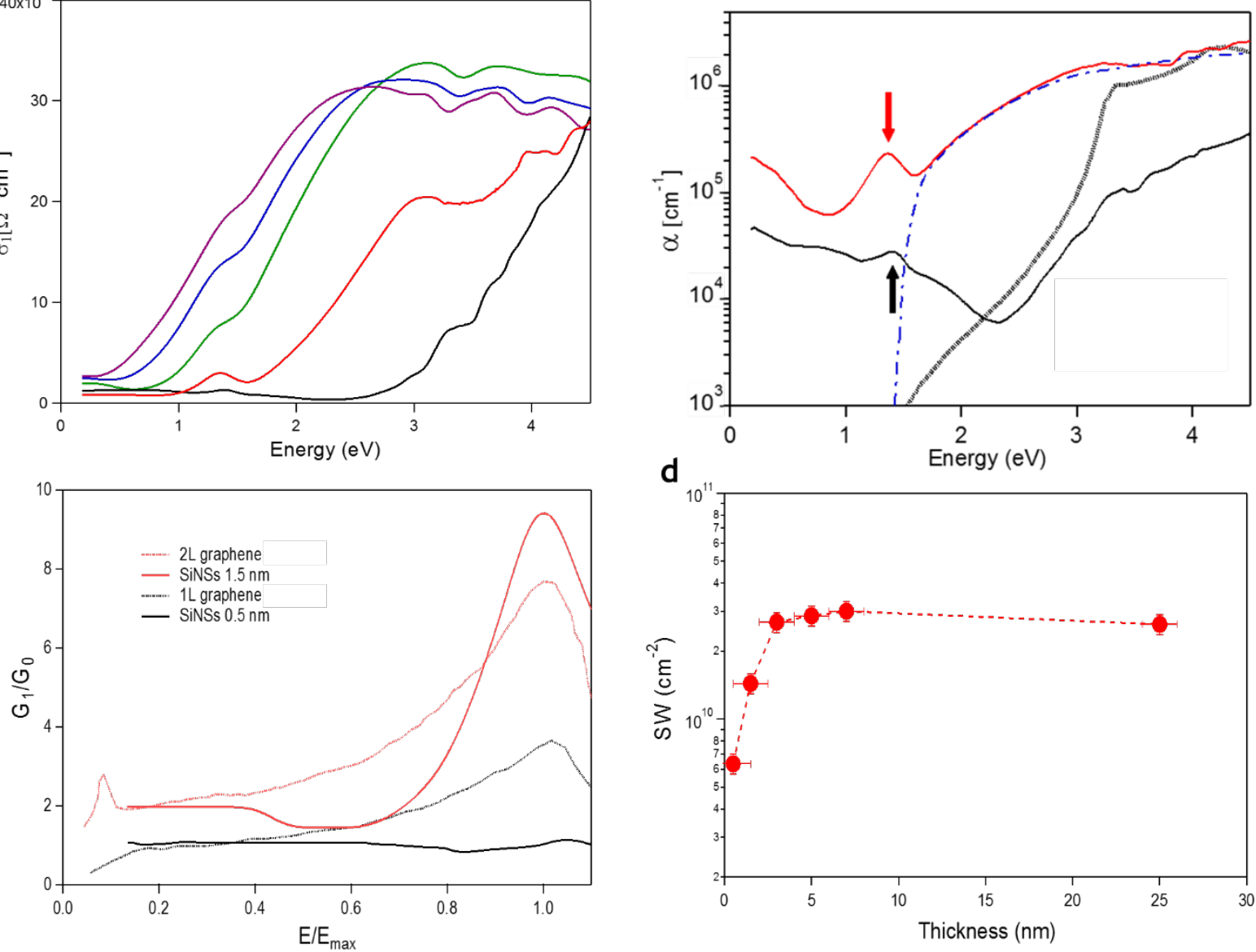

d

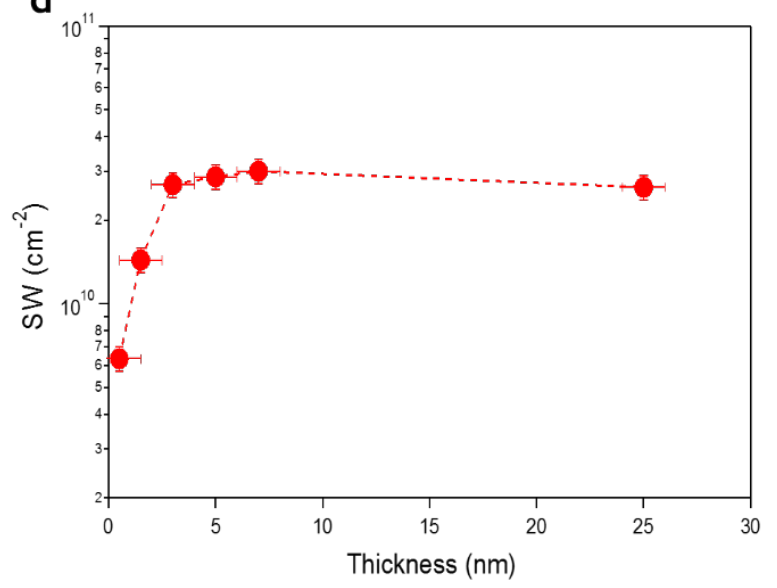

Figure 3. (a) The real part of the optical conductivity $\sigma_{1}(\omega)$ for the five thickness scrutinized: 0.5 (black), 1.5 (red), 3 (green), 5 (blue), and $7 \mathrm{~nm}$ (purple). (b) The absorption coefficient $\alpha(\omega)$ for the 0.5 and $1.5 \mathrm{~nm}$-thick film (black and red curves), of the $25 \mathrm{~nm}$-thick bulk reference (dashed-dotted blue line) and that of crystalline bulk silicon (dashed gray line). Black and red arrows point out the transition I for 0.5 and $1.5 \mathrm{~nm}$, respectively. At $25 \mathrm{~nm}$, one nearly recovers the bulk properties of 3D silicon. (c) The real part of the optical conductance $\mathrm{G}_{1}(\omega)$ normalized to the universal optical conductance $\mathrm{G}_{0}$ for the 0.5 (black line) and $1.5 \mathrm{~nm}$ (red line) thickness SiNSs. In order to properly compare these spectra with those of single layer (black dotted line) and two layers (red dotted line) graphene, the frequency axis is normalized to the bonding-antibonding $\pi$ transition appearing around $1.4 \mathrm{eV}$ in SiNSs and around $4.6 \mathrm{eV}$ in graphene. ${ }^{23}$ (d) $\mathrm{SW}$ calculated from $\omega_{\mathrm{m}}=0.25$ to $\omega_{\mathrm{M}}=4.5 \mathrm{eV}$ for the five thicknesses scrutinized in a and reference $25 \mathrm{~nm}$-thick sample (red dashed line is a guide for the eye). 
In the visible spectral range, $\sigma_{1}(\omega)$ drops with a broad gap and rises linearly around $3 \mathrm{eV}$ up to the $\mathrm{UV}$ range. Interestingly, the spectral feature around $1.4 \mathrm{eV}$ and the increasing absorption around $4 \mathrm{eV}$, closely resemble those arising from I and II interband transitions in freestanding silicene by $a b$ initio calculation. ${ }^{6-8}$ When increasing the SiNS thickness to $1.5 \mathrm{~nm}$ (red curve in Figure 3a), $\sigma_{1}(\omega)$ is still nearly flat below $1 \mathrm{eV}$, the absorption peak around $1.4 \mathrm{eV}$ is now very well defined and the linearly increasing absorption starts around 2 $\mathrm{eV}$. A close inspection of Figure 3a clearly evidences that the absorption feature around $1.4 \mathrm{eV}$ is reminiscent of the calculated peak I appearing at about $1.6 \mathrm{eV}$ in the freestanding silicene (see below). ${ }^{6-8}$ For a further increase of the thickness, one observes a softening and broadening of the UV absorption which progressively superimposes to the peak around $1.4 \mathrm{eV}$. The flat absorption region, although still visible up to the maximum thickness of $7 \mathrm{~nm}$, is more and more reduced to the low-frequency part of the conductivity spectrum. In order to investigate the evolution of the optical properties of SiNSs samples with thickness and to recover the expected optical behavior of bulk silicon, Figure 3b compares the absorption coefficient $\alpha(\omega)$ of the 0.5 and $1.5 \mathrm{~nm}$-thick SiNSs (black and red curves), of the reference amorphous $25 \mathrm{~nm}$-thick sample (blue dasheddotted curve), and of bulk crystalline silicon (dashed-line black curve) ${ }^{24}$ At first glance, we notice that the optical behavior of SiNSs strikingly deviates from that of a bulk silicon whose absorption is partially restored only for the thickness of $25 \mathrm{~nm}$ with a well-defined band gap around $1.3 \mathrm{eV}$. The finite absorption in the IR region is therefore strongly indicative of an exotic electronic structure reminiscent of the massless Dirac fermions. Although not as direct evidence as provided by other experimental techniques, e.g. angle-resolved photoelectron spectroscopy, we notice the sensitivity of the optical conductivity is inherently related to the JDOS, thus bringing to a reliable evidence of the electronic structure of the SiNSs. Indeed, similar to graphene, the optical conductivity over the corresponding spectral range can be demonstrated to be a robust quantity. ${ }^{25}$ In particular, not only all the $\sigma_{1}(\omega)$ spectra reported here (up to $7 \mathrm{~nm}$-thick SiNSs) markedly differ from the absorbance spectrum of cubic diamond bulk silicon, but also from that of silicite (the surmised silicon counterpart of graphite), or other reported silicon allotropes. ${ }^{26-28}$ Nonetheless, such a different behavior can be explained by the stabilization of a $2 \mathrm{D}$ hexagonal phase of silicon in the early stage of the epitaxy on the $\mathrm{Al}_{2} \mathrm{O}_{3}(0001)$ substrate, which consequently affects the subsequent three-dimensional (3D) growth regime. This argument is further corroborated by the comparison with graphene. Figure $3 \mathbf{c}$ shows the low-frequency behavior of SiNSs ( 0.5 and $1.5 \mathrm{~nm}$-thick), described in terms of the real part of the optical conductance $\mathrm{G}_{1}(\omega)$. 
This quantity, which is related to the optical conductivity through the equation $G_{1}(\omega)=\sigma_{1}(\omega) \cdot d$, where $d$ is the film thickness, is conventionally used to describe the optical properties of $2 \mathrm{D}$ materials like graphene ${ }^{25}$ and topological insulators. ${ }^{29,30}$ In Figure $3 \mathbf{c} \mathrm{G}_{1}(\omega)$ is derived and then normalized to the universal conductance $\mathrm{G}_{0} \equiv$ $\mathrm{e}^{2} / 4 \hbar,{ }^{31}$ which is an intrinsic property of $2 \mathrm{D}$ massless electrons as experimentally measured in graphene and confirmed by theoretical predictions. ${ }^{23,32}$ Moreover, in order to facilitate the comparison with graphene, a universal frequency axis is obtained by normalizing the actual frequency with respect to the bondingantibonding $\pi$ transition appearing around $1.4 \mathrm{eV}$ in SiNSs and around $4.6 \mathrm{eV}$ in graphene, respectively. ${ }^{23} \mathrm{As}$ observed in Figure 3c, $\mathrm{G}_{1} / \mathrm{G}_{0}$ below $1 \mathrm{eV}$ saturates (as a flat background) to the universal value 1 and 2 for $\mathrm{d}=0.5$ and $1.5 \mathrm{~nm}$, respectively. This is in good agreement with measurements reported on the single- and twolayers graphene (see for instance Ref. $^{23}$ ) and represented in the same figure by black- and red-dotted curves, respectively. The normalized optical conductance spectra of SiNSs in the lowest photon energies look comparatively flatter than those of graphene due to the absence of doping (sample-dependent) issues. ${ }^{25}$ This universal scaling behavior, i.e. an integer value of universal conductance as a function of the layer thickness, is a characteristic hallmark of $2 \mathrm{D}$ Dirac fermions in graphene, and suggests that $2 \mathrm{D}$ silicon grown on a $\mathrm{Al}_{2} \mathrm{O}_{3}(0001)$ substrate retains the properties of freestanding silicene, whose low-energy electrodynamics is related to a linear (Dirac-like) electronic dispersion. Furthermore, by comparison with graphene (Figure 3c), we can speculate that 0.5 and $1.5 \mathrm{~nm}$-thick SiNSs may be regarded as if they were single and double-layers silicene, respectively. Additional experimental and theoretical efforts are therefore highly demanded to unravel this surmise.

Finally, we study the redistribution of the optical spectral weight (SW) using the $f$-sum rule analysis. Figure 3d shows the experimental $S W=\frac{120}{\pi} \int_{\omega_{m}}^{\omega_{M}} \sigma_{1}(\omega) d \omega$ for a frequency range between $\omega_{\mathrm{m}}=0.25$ and $\omega_{\mathrm{M}}=4.5 \mathrm{eV}$ for all the SiNSs thicknesses considered. The SW plot exhibits a twofold fashion, namely a steep monotonic increase with thickness up to $\sim 3 \mathrm{~nm}$ and then a nearly flat behavior for thicker SiNSs, with saturation at $\sim 25$ $\mathrm{nm}$ (amorphous silicon reference), indicative of a 2D-to-3D crossover. Although other measurements are necessary to elucidate the transition from 2D silicene-like to 3D silicon-like optical behavior, the thickness range here considered can offer a great potential for applications beyond the state-of-the-art on IR-visible 
photonics and optoelectronics based on dimensionally reduced silicon, where a unique benefit is gained from silicon being sandwiched in between transparent substrate and capping layer.

Although the experimental data for the thinnest SiNSs surveyed show a fair agreement with the theoretical predictions of freestanding silicene, it is demanding to unravel the role of the $\mathrm{Al}_{2} \mathrm{O}_{3}(0001)$ substrate, in consideration of the strong influence of the substrate in the optical properties of other silicene systems, e.g. silicene supported by $\operatorname{Ag}(111) .{ }^{11}$ Moreover, the observed experimental data are apparently not compatible with the calculated electronic bandstructure of the supported silicene proposed by Chen et al., which actually reveals that the Dirac cone disappears, and just a vague reminiscence of it survives at the $\mathrm{K}$ point of the BZ with a bandgap of $0.44 \mathrm{eV} \cdot{ }^{13}$ Hence, in order to elucidate the experimental scenario, we performed ab initio calculations of the structural, electronic, and optical properties of silicene on $\mathrm{Al}_{2} \mathrm{O}_{3}(0001)$, thus focusing on the comparison with the thinnest (CT) SiNS. We theoretically deposit a single layer of silicon atoms on the surface, exploring different possible adsorption geometries (see Supporting Information Figures S7 and S8). The $\mathrm{Al}_{2} \mathrm{O}_{3}(0001)$ surface periodicity is $3 \times 3$ with respect to the clean one, whereas the silicon overlayer's periodicity becomes $\sqrt{ } 13 \times \sqrt{ } 13 \mathrm{R} 13.9^{\circ}$ with respect to the ideal freestanding silicene. The Born-Oppenheimer energy surface of the so-composed system, i.e. silicene and $\mathrm{Al}_{2} \mathrm{O}_{3}(0001)$, presents many local minima. Several different and metastable $\sqrt{13} \times \sqrt{13}$ silicene geometries on the $3 \times 3 \mathrm{Al}_{2} \mathrm{O}_{3}(0001)$ substrate were indeed found in addition to the structures modeled by Chen et al. ${ }^{13}$ Differences among them rely on the mutual position of silicon and aluminum/oxygen atoms. In all the cases, the silicene overlayer is subjected to a strain of about $3.3 \%$ because of the lattice mismatch with the substrate and also silicene loses its pristine $\mathrm{D}_{3 \mathrm{~d}}$ symmetry. Basically, all the studied geometries can be grouped into two categories, where the subsystems $\mathrm{Al}_{2} \mathrm{O}_{3}(0001)$ and silicene are either strongly interacting (with average distance between the substrate and the overlayer of about $2.8 \AA$ ), or weakly interacting (with an average distance of about $3.3 \AA$ ). Table S2 summarizes the main properties of the two representative cases, the SIS (strongly-interacting silicene, belonging to the former group) and the WIS (weakly-interacting silicene, belonging to the latter group). The energy differences among the reported geometries are small, hence their coexistence is possible even at room temperature. Even if the binding energy is moderate, indicating a quite limited interaction with the substrate, a gap as large as $0.44 \mathrm{eV}$ opens at $\mathrm{K}$ point of $\mathrm{BZ}$ in the case of the SIS configuration, in agreement with the results by Chen et al. ${ }^{13}$ The 
Dirac cone is affected being hardly identifiable, as shown in Figure 4a, where the calculated electronic band structure is reported in the $\mathrm{BZ}$ of the $\sqrt{ } 13 \times \sqrt{ } 13$ lattice.
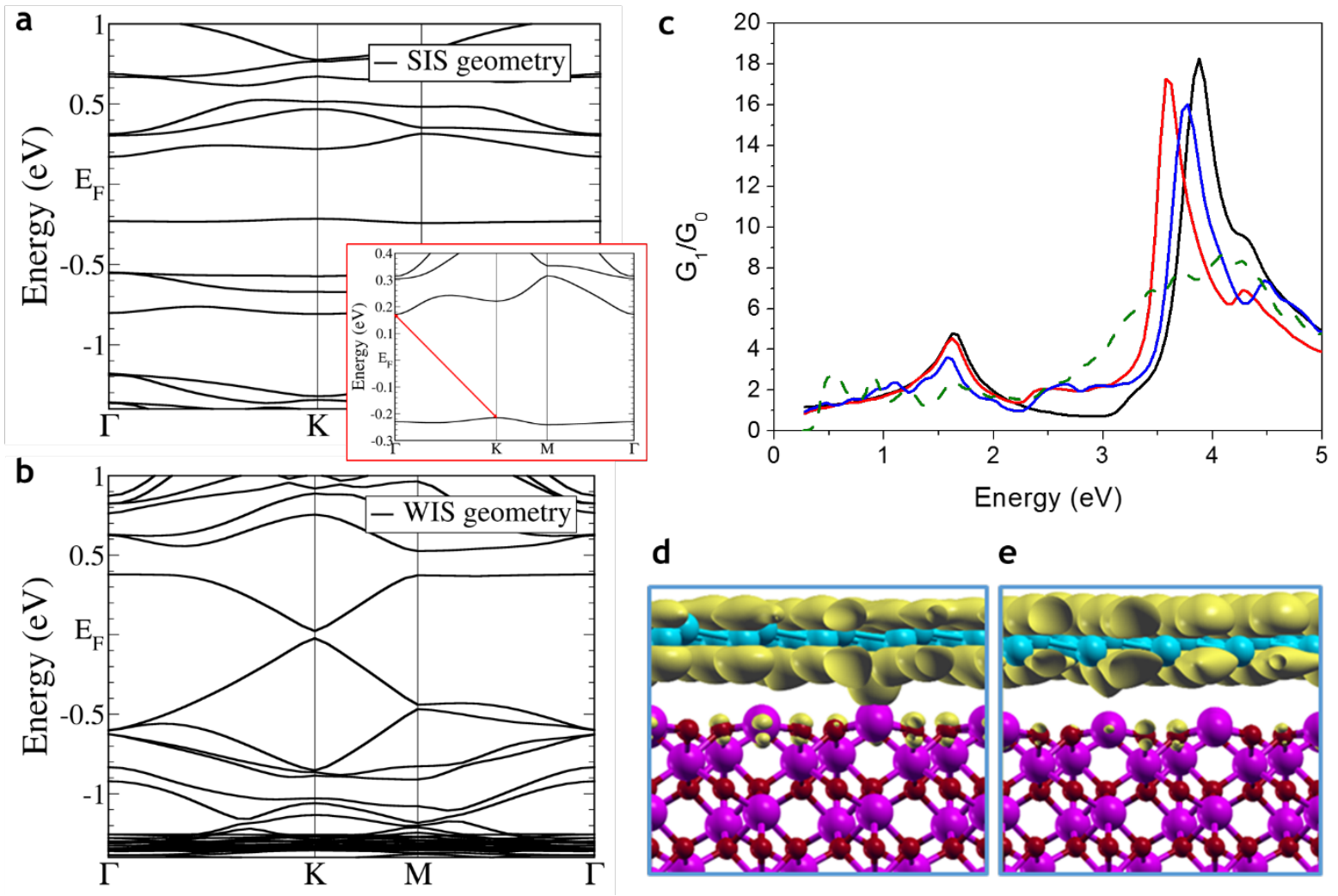

Figure 4. (a) Calculated electronic band structure for SIS geometry (inset shows zoom of the indirect bandgap) and (b) for the WIS geometry. (c) Normalized conductance $\mathrm{G}_{1} / \mathrm{G}_{0}$ for SIS (green dashed) and WIS (blue) on $\mathrm{Al}_{2} \mathrm{O}_{3}(0001)$ configurations. Corresponding quantities for ideal freestanding silicene (black) and for freestanding silicene with 3\% strain (red) are also reported for comparison. (d) Squared modulus of the electronic wavefunctions at the highest occupied bands and (e) at the lowest unoccupied bands at the K point for the WIS configuration.

On the contrary, when the interaction is smaller, as in the WIS geometry, the Dirac cone still survives but the Dirac fermions become massive. In fact, as shown in Figure $\mathbf{4 b}$, a small gap of about $0.05 \mathrm{eV}$ opens at K point but a significant similarity with the linear band behavior of freestanding silicene close to the Fermi level still survives. In other words, the degree of interaction of the silicon overlayer with $\mathrm{Al}_{2} \mathrm{O}_{3}(0001)$ tunes the electronic band structures which, as we will show in the following, have profound consequences on the low-energy part of optical spectra of the system. $\mathrm{Al}_{2} \mathrm{O}_{3}(0001)$ surface possesses a DFT gap of about $5 \mathrm{eV}$, hence it is transparent 
in the energy range where instead ideal silicene absorbs. From the two representative geometries WIS and SIS, the optical properties of silicene on $\mathrm{Al}_{2} \mathrm{O}_{3}(0001)$ have been calculated in terms of $\mathrm{G}_{1}(\omega)$ (Figure $4 \mathbf{c}$ and Supporting Information) and compared to that of the ideal system. For the SIS investigated geometry the lowenergy optical conductivity (green dashed line, Figure 4c) strongly differs from that of freestanding silicene (black line), due to the opening of a significant gap in the mid-IR (about $0.5 \mathrm{eV}$ ), which has not been observed in the experimental data, and a no discernible peak I. On the other hand, the WIS geometry (blue line) shows optical properties that are similar to those of ideal silicene and qualitatively comparable to the experimental data of the CT sample (Figure 3a). In particular, the WIS configuration still shows a discernible peak I slightly softened with respect to the freestanding silicene (the same softening trend has been observed experimentally) and a nearly flat conductivity below the peak I. Strain effects due to the lattice mismatch between silicene and $\mathrm{Al}_{2} \mathrm{O}_{3}(0001)$ in the weak-interacting configuration are responsible of the small shift in the peak II, which in the unstrained silicene (black curve in Figure 4c) is at $3.9 \mathrm{eV}$ (due to the interband $\sigma \rightarrow \sigma^{*}$ transition at $\Gamma$ ), in artificially strained freestanding silicene appears at $3.6 \mathrm{eV}$ (red curve), and in the WIS appears at $3.8 \mathrm{eV}$ (blue line). Also, the spectrum in the energy region between 2.2 and $3.0 \mathrm{eV}$ clearly shows similarities with the strained freestanding silicene. The peak I around $1.6 \mathrm{eV}$ is almost unaffected by the presence of the substrate and by the strain, and is very close to the experimentally observed one. At lower-energies, the normalized conductance $\mathrm{G}_{1} / \mathrm{G}_{0}$ of the WIS structure, still related to low-energy $\pi \rightarrow \pi^{*}$ transitions but close to K point, tends to 1 as occurs for ideal silicene and the CT sample (Figure 3c). The squared modulus of the wavefunction at $\mathrm{K}$ point for the highest occupied band (Figure 4d) and the lowest unoccupied band (Figure 4e) are essentially due to silicon, indicating that silicon is atomically bonded to but almost electronically decoupled from the substrate. Although the WIS structure significantly helps to understand the conductivity spectrum of the supported silicene on $\mathrm{Al}_{2} \mathrm{O}_{3}(0001)$, especially in comparison with the experimental data of SiNSs at the 2D limit on the same substrate, it is not clear yet why the WIS should set in instead of the other energetically competing configuration. Bearing in mind that the energy difference between the SIS and WIS structures is not large, a possible argument for the stabilization of the latter relies on the high-temperature growth condition $\left(670{ }^{\circ} \mathrm{C}\right.$, see Methods) as promoter for the growth of a silicene configuration with a relatively lower-stability. By and large, we speculate that as much as the interaction between (reconstructed) silicene and $\mathrm{Al}_{2} \mathrm{O}_{3}(0001)$ is relaxed, the theoretical electronic and optical descriptions turn out to improve the agreement with the 
experimental data. Our work is anyway demanding for further specific investigation devoted to understand the possible presence of a buffer layer decreasing the interaction between silicon and substrate, and to further explore the manifold configurations in which SiNSs may accommodate on $\mathrm{Al}_{2} \mathrm{O}_{3}(0001)$.

Summarizing, we investigated the optical properties of silicon at the $2 \mathrm{D}$ limit on $\mathrm{Al}_{2} \mathrm{O}_{3}(0001)$ by fabricating and encapsulating specific SiNSs with spatially constant and variable thickness. In this way, a Dirac-like behavior is observed in the IR part of the optical conductivity spectra of the 2D SiNSs, thus suggesting the presence of Dirac fermions hosted by a silicene-like structure. This argument is supported by the following evidences. First, the observed $\sigma_{1}(\omega)$ of the CT sample shows an overall behavior similar to that expected from the ideal silicene with a clear $\pi \rightarrow \pi^{*}$ interband transition feature. Second, the quantized conductance depending on the silicon thickness further suggests that the SiNSs at the 2D limit possess silicene-like properties, as already proved for graphene. Third, this experimental scenario is consistent with the $a b$ initio model of a $\sqrt{ } 13 \times \sqrt{ } 13$ structured silicene that is weakly interacting with the substrate. The identification of a Dirac-like optical conductivity peaked in the near-IR regime from a 2D silicon grown on an optical transparent substrate opens unexplored avenues in establishing a silicene-based photonics. In perspective, the proposed methodology can be further extended to other (also heavier) X-enes, paving the way to the exploitation of their exotic properties related to the non-trivial topology for applications in the optoelectronics and photonics fields, especially for the silicon case, in light of the well-known long lasting expertise on the silicon on sapphire to provide reliable multifunctional devices.

\section{Methods}

SiNSs were grown in a UHV chamber (base pressure $10^{-10}$ mbar) system equipped with interconnected chambers for sample growth via MBE and chemical analysis via XPS. Several hours degassing at $250{ }^{\circ} \mathrm{C}$ was performed on the single side polished $\mathrm{Al}_{2} \mathrm{O}_{3}(0001)$ samples $\left(\right.$ Crystec $\left.{ }^{\circledR}\right)$ before silicon growth. SiNSs were deposited from a heated crucible in the built-in evaporator or from a piece of silicon wafer with a rate of $\sim 1$ $\mathrm{nm} /$ hour at substrate temperature of $670{ }^{\circ} \mathrm{C}$ and compared with a reference $\sim 25 \mathrm{~nm}$-thick amorphous sample grown at room temperature. Temperature reading was crosschecked by pyrometer-based calibration of the thermocouple attached under the sample holder and the deposition rate was confirmed ex situ by means of AFM thickness measurements. AFM investigations were performed ex situ on the capped samples by means 
of an AFM-Bruker ${ }^{\circledR}$ system operating in tapping mode and equipped with ultra-sharp silicon probe (tip diameter $<10 \mathrm{~nm}$ ). XPS characterization was carried out by means of a non-monochromatized $\mathrm{Mg}$ and $\mathrm{Al} \mathrm{K}_{\alpha}$ sources (1253.6 and $1486.6 \mathrm{eV}$, respectively) at a take-off angle of $37^{\circ}$. Adventitious $\mathrm{C} 1 \mathrm{~s}$ binding energy at $285.0 \mathrm{eV}$ was used as reference to calibrate the energy shift of core levels due to substrate-induced charging effects. Amorphous $5 \mathrm{~nm}$-thick $\mathrm{Al}_{2} \mathrm{O}_{3}$ capping layer was grown in situ through reactive co-deposition. ${ }^{16} E x$ situ Raman spectroscopy was performed by using a Renishaw ${ }^{\circledR}$ Invia spectrometer equipped with the 2.54 $\mathrm{eV} / 488 \mathrm{~nm}$ line of an $\mathrm{Ar}^{+}$laser line focused on the sample by a $100 \times$ Leica objective ( 0.9 numerical aperture) providing a spot diameter of about $0.4 \mu \mathrm{m}$. The power at the sample was maintained below $5 \mathrm{~mW}$ in order to prevent laser induced sample heating. All the measurements were carried out in a z-backscattering geometry. For the TEM studies, lamellae were covered with metal (chromium) deposition to protect them during the procedure and prepared using focus ion beam. The lamella was cut out by milling with $30 \mathrm{kV}$ gallium ions and thinned down with subsequent steps of 30 and $5 \mathrm{kV}$ ion milling and then mechanically transferred to a copper TEM grid. Scanning TEM (STEM) analyses were conducted using an aberration-corrected TEM operated at $200 \mathrm{kV}$. For the chemical analysis, EDX measurements were carried out using the same microscope equipped with a $80 \mathrm{~mm}^{2}$ EDX silicon drift detector. In addition to EDX, the cross-sectional uniformity of the SiNSs has also been verified by Raman spectroscopy and IR microscopy with sampling steps of $0.2 \mathrm{~cm}$ and $100 \mu \mathrm{m}$, respectively. In order to investigate the optical properties of SiNSs grown on $\mathrm{Al}_{2} \mathrm{O}_{3}(0001)$, we measured the absolute transmittance $\mathrm{T}(\omega)$ on both type of samples, i.e. constant-thickness $(\mathrm{CT}, 0.5 \mathrm{~nm})$, variable-thickness (VT, from 1.5 to $7 \mathrm{~nm}$ ), and reference $25 \mathrm{~nm}$-thick amorphous film, in the frequency $(\omega)$ range from 0.25 to $4.5 \mathrm{eV}$ by using a Michelson IFS66V Bruker ${ }^{\circledR}$ interferometer in the IR and a JASCO ${ }^{\circledR}$ spectrometer in the visible/UV region. From the measured T( $\omega)$ (see Figure S6 of Supporting Information), we have derived the optical conductivity $\sigma(\omega)=\sigma_{1}(\omega)+i \sigma_{2}(\omega)$ of SiNSs through the use of the Reffit program by means of KramersKronig transformations, which takes into account the stratified structure of samples..$^{33} \sigma_{1}(\omega)$ has been obtained by considering the actual complex refractive index of $\mathrm{Al}_{2} \mathrm{O}_{3}(0001)$ as determined from its absolute transmittance measured for a bare substrate coming from the same batch. The optical conductivity as extracted through Reffit from $T(\omega)$ by considering a two layers system (substrate and film) or three layers system (substrate, film, and amorphous capping layer) does not depend of the presence of the capping layer. 
The electronic and optical properties of silicon on $\mathrm{Al}_{2} \mathrm{O}_{3}(0001)$ were calculated within ab initio DFT using norm-conserving pseudopotential with a Perdew-Burke-Ernzerhof exchange and correlation potential in the Quantum Espresso code. ${ }^{34}$ The $\mathrm{Al}_{2} \mathrm{O} 3(0001)$ substrate was simulated with a symmetric slab made of 18 layers relaxed using $3 \times 3 \times 1$ k-points and an energy cutoff of $65 \mathrm{Ry}$. Silicene was added on the top $\mathrm{Al}_{2} \mathrm{O}_{3}(0001)$ surface and relaxed with the inclusion of van der Waals forces. A vacuum of $14 \AA$ ensured that periodic images of the slab do not interact. Optical spectra were calculated within the single particle approximation (Fermi golden rule) using $18 \times 18 \times 1 \mathrm{k}$-points for the $\sqrt{ } 13 \times \sqrt{ } 13 \mathrm{R} 13.9^{\circ}$ silicene on substrate and $400 \times 400 \times 1 \mathrm{k}$-points for $1 \times 1$ freestanding silicene.

\section{Associated Content}

The Supporting Information is available free of charge on the ACS Publications website at DOI:

Additional data on VT, CT, and amorphous reference samples (Figures S1, S2, and S3), XPS data (Table S1), EDX maps (Figure S4), real and imaginary parts of the $\mathrm{Al}_{2} \mathrm{O}_{3}(0001)$ refraction index (Figure S5), optical transmittance of SiNSs (Figure S6), structural models of SIS and WIS configurations (Figure S7 and S8) and their properties (Table S2), and supporting text on the calculation of the optical properties.

\section{Author Information}

Corresponding Authors

*E-mail: Prof. Stefano Lupi (stefano.lupi@roma1.infn.it).

*E-mail: Dr. Alessandro Molle (alessandro.molle@mdm.imm.cnr.it)

\section{ORCID}

Carlo Grazianetti: 0000-0003-0060-9804

Christian Martella: 0000-0003-1811-165X

Paola Gori: 0000-0001-6896-5560

Olivia Pulci: 0000-0002-9725-487X

Alessandro Molle: 0000-0002-3860-4120 
Stefano Lupi: 0000-0001-7002-337X

Author Contributions

C.G. developed the epitaxial growth of the SiNSs samples and subsequent $\mathrm{Al}_{2} \mathrm{O}_{3}$ encapsulation. C.G. also performed XPS analysis. C.M. conducted Raman spectroscopy and AFM studies of all the samples. P.T. and D.G. carried out TEM and EDX investigations. S.D. and S.L. performed and elaborated transmittance measurements. O.P. and P.G. carried out the theoretical investigation and related electronic and optical models. C.G., A.M., and S.L. planned the experiments. All authors contributed to the writing based on the draft written by C.G. A.M. and S.L. coordinated and supervised the research.

Notes

The authors declare no competing financial interest.

\section{Acknowledgments}

Authors acknowledge M. Alia (CNR-IMM) for technical support and Flavio Giorgianni (SwissFEL, Paul Scherrer Institute) for support in the optical transmittance measurements; A.M. acknowledges funding support from H2020 ERC CoG 2017 Grant N. 772261 “XFab”. A.M. and C.G. acknowledge funding support from CNR grant Laboratori Congiunti “SFET”, and Fondazione CARIPLO - Regione Lombardia for the project “Crystel”, grant N. 2016-0978. O.P. acknowledges funding support from HORIZON2020 MSCA RISE EU project "CoExAN" (GA644076). O.P. and P.G. acknowledge CINECA HPC center and CRESCO HPC center for having granted CPU time.

\section{References}

(1) Ferrari, A. C.; Bonaccorso, F.; Fal'ko, V.; Novoselov, K. S.; Roche, S.; Bøggild, P.; Borini, S.; Koppens, F. H. L.; Palermo, V.; Pugno, N.; et al. Science and Technology Roadmap for Graphene, Related Two-Dimensional Crystals, and Hybrid Systems. Nanoscale 2015, 7 (11), 4598-4810.

(2) Atabaki, A. H.; Moazeni, S.; Pavanello, F.; Gevorgyan, H.; Notaros, J.; Alloatti, L.; Wade, M. T.; Sun, C.; Kruger, S. A.; Meng, H.; et al. Integrating Photonics with Silicon Nanoelectronics for the next Generation of Systems on a Chip. Nature 2018, 556 (7701), 349-354.

(3) Grazianetti, C.; Cinquanta, E.; Molle, A. Two-Dimensional Silicon: The Advent of Silicene. 2D 
Mater. 2016, 3 (1), 012001.

(4) Molle, A.; Goldberger, J.; Houssa, M.; Xu, Y.; Zhang, S. C.; Akinwande, D. Buckled Two-

Dimensional Xene Sheets. Nature Materials. Nature Research January 16, 2017, pp 163-169.

(5) Castellanos-Gomez, A. Why All the Fuss about 2D Semiconductors? Nat. Photonics 2016, 10 (4), 202-204.

(6) Bechstedt, F.; Matthes, L.; Gori, P.; Pulci, O. Infrared Absorbance of Silicene and Germanene. Appl. Phys. Lett. 2012, 100 (26), 261906.

(7) Matthes, L.; Gori, P.; Pulci, O.; Bechstedt, F. Universal Infrared Absorbance of Two-Dimensional Honeycomb Group-IV Crystals. Phys. Rev. B 2013, 87 (3), 035438.

(8) Matthes, L.; Pulci, O.; Bechstedt, F. Optical Properties of Two-Dimensional Honeycomb Crystals Graphene, Silicene, Germanene, and Tinene from First Principles. New J. Phys. 2014, 16 (10), 105007.

(9) Gori, P.; Pulci, O.; Ronci, F.; Colonna, S.; Bechstedt, F. Origin of Dirac-Cone-like Features in Silicon Structures on $\operatorname{Ag}(111)$ and $\operatorname{Ag}(110)$. J. Appl. Phys. 2013, 114 (11), 113710.

(10) Hogan, C.; Pulci, O.; Gori, P.; Bechstedt, F.; Martin, D. S.; Barritt, E. E.; Curcella, A.; Prevot, G.; Borensztein, Y. Optical Properties of Silicene, $\mathrm{Si} / \operatorname{Ag}(111)$, and $\mathrm{Si} / \operatorname{Ag}(110)$. Phys. Rev. B 2018, 97 (19), 195407.

(11) Cinquanta, E.; Fratesi, G.; dal Conte, S.; Grazianetti, C.; Scotognella, F.; Stagira, S.; Vozzi, C.; Onida, G.; Molle, A. Optical Response and Ultrafast Carrier Dynamics of the Silicene-Silver Interface. Phys. Rev. B 2015, 92 (16), 165427.

(12) French, R. H. Electronic Band Structure of A12O3, with Comparison to Alon and AIN. J. Am. Ceram. Soc. 1990, 73 (3), 477-489.

(13) Chen, M. X.; Zhong, Z.; Weinert, M. Designing Substrates for Silicene and Germanene: FirstPrinciples Calculations. Phys. Rev. B 2016, 94 (7), 075409.

(14) Ahn, J.; Rabalais, J. W. Composition and Structure of the Al2O3\{0001\}-(1 × 1) Surface. Surf. Sci. 1997, $388(1-3), 121-131$.

(15) Eng, P. J.; Trainor, T. P.; Brown, G. E.; Waychunas, G. A.; Newville, M.; Sutton, S. R.; Rivers, M. L. Structure of the Hydrated $\alpha$-A12O3(0001) Surface. Science (80-. ). 2000, 288 (5468), 1029-1033. 
(16) Molle, A.; Grazianetti, C.; Chiappe, D.; Cinquanta, E.; Cianci, E.; Tallarida, G.; Fanciulli, M. Hindering the Oxidation of Silicene with Non-Reactive Encapsulation. Adv. Funct. Mater. 2013, 23 (35), 4340-4344.

(17) Molle, A.; Lamperti, A.; Rotta, D.; Fanciulli, M.; Cinquanta, E.; Grazianetti, C. Electron Confinement at the Si/MoS2 Heterosheet Interface. Adv. Mater. Interfaces 2016, 3 (10), 1500619.

(18) Friedlein, R.; Fleurence, A.; Aoyagi, K.; de Jong, M. P.; Van Bui, H.; Wiggers, F. B.; Yoshimoto, S.; Koitaya, T.; Shimizu, S.; Noritake, H.; et al. Core Level Excitations-A Fingerprint of Structural and Electronic Properties of Epitaxial Silicene. J. Chem. Phys. 2014, 140 (18), 184704.

(19) Baba, Y.; Shimoyama, I.; Hirao, N.; Sekiguchi, T. Structures of Quasi-Freestanding Ultra-Thin Silicon Films Deposited on Chemically Inert Surfaces. Chem. Phys. 2014, 444, 1-6.

(20) Grazianetti, C.; Cinquanta, E.; Tao, L.; De Padova, P.; Quaresima, C.; Ottaviani, C.; Akinwande, D.; Molle, A. Silicon Nanosheets: Crossover between Multilayer Silicene and Diamond-like Growth Regime. ACS Nano 2017, 11 (3), 3376-3382.

(21) Cinquanta, E.; Scalise, E.; Chiappe, D.; Grazianetti, C.; Van Den Broek, B.; Houssa, M.; Fanciulli, M.; Molle, A. Getting through the Nature of Silicene: An Sp2-Sp3 Two-Dimensional Silicon Nanosheet. J. Phys. Chem. C 2013, 117 (32), 16719-16724.

(22) Nakamura, R.; Ishimaru, M.; Yasuda, H.; Nakajima, H. Atomic Rearrangements in Amorphous Al 2 O 3 under Electron-Beam Irradiation. J. Appl. Phys. 2013, 113 (10), 1649-91901.

(23) Mak, K. F.; Shan, J.; Heinz, T. F. Seeing Many-Body Effects in Single- and Few-Layer Graphene: Observation of Two-Dimensional Saddle-Point Excitons. Phys. Rev. Lett. 2011, 106 (4), 046401.

(24) Jovanov, V.; Ivanchev, J.; Knipp, D. Standing Wave Spectrometer. Opt. Express 2010, 18 (2), 426.

(25) Mak, K. F.; Sfeir, M. Y.; Wu, Y.; Lui, C. H.; Misewich, J. A.; Heinz, T. F. Measurement of the Optical Conductivity of Graphene. Phys. Rev. Lett. 2008, 101 (19), 196405.

(26) Cahangirov, S.; Özçelik, V. O.; Rubio, A.; Ciraci, S. Silicite: The Layered Allotrope of Silicon. Phys. Rev. B 2014, 90 (8), 085426.

(27) Botti, S.; Flores-Livas, J. A.; Amsler, M.; Goedecker, S.; Marques, M. A. L. Low-Energy Silicon Allotropes with Strong Absorption in the Visible for Photovoltaic Applications. Phys. Rev. B Condens. Matter Mater. Phys. 2012, 86 (12), 121204. 
(28) Kim, D. Y.; Stefanoski, S.; Kurakevych, O. O.; Strobel, T. A. Synthesis of an Open-Framework Allotrope of Silicon. Nat. Mater. 2014, 14 (2), 169-173.

(29) Di Pietro, P.; Vitucci, F. M.; Nicoletti, D.; Baldassarre, L.; Calvani, P.; Cava, R.; Hor, Y. S.; Schade, U.; Lupi, S. Optical Conductivity of Bismuth-Based Topological Insulators. Phys. Rev. B 2012, 86 (4), 045439.

(30) Valdés Aguilar, R.; Stier, A. V.; Liu, W.; Bilbro, L. S.; George, D. K.; Bansal, N.; Wu, L.; Cerne, J.; Markelz, A. G.; Oh, S.; et al. Terahertz Response and Colossal Kerr Rotation from the Surface States of the Topological Insulator Bi 2 Se 3. Phys. Rev. Lett. 2012, 108 (8), 087403.

(31) Kuzmenko, A. B.; van Heumen, E.; Carbone, F.; van der Marel, D. Universal Optical Conductance of Graphite. Phys. Rev. Lett. 2008, 100 (11), 117401.

(32) Min, H.; MacDonald, A. H. Origin of Universal Optical Conductivity and Optical Stacking Sequence Identification in Multilayer Graphene. Phys. Rev. Lett. 2009, 103 (6).

(33) Kuzmenko, A. B. Kramers-Kronig Constrained Variational Analysis of Optical Spectra. Rev. Sci. Instrum. 2005, $76(8), 083108$.

(34) Giannozzi, P.; Baroni, S.; Bonini, N.; Calandra, M.; Car, R.; Cavazzoni, C.; Ceresoli, D.; Chiarotti, G. L.; Cococcioni, M.; Dabo, I.; et al. QUANTUM ESPRESSO: A Modular and Open-Source Software Project for Quantum Simulations of Materials. J. Phys. Condens. Matter 2009, 21 (39), 395502.

\section{TOC}

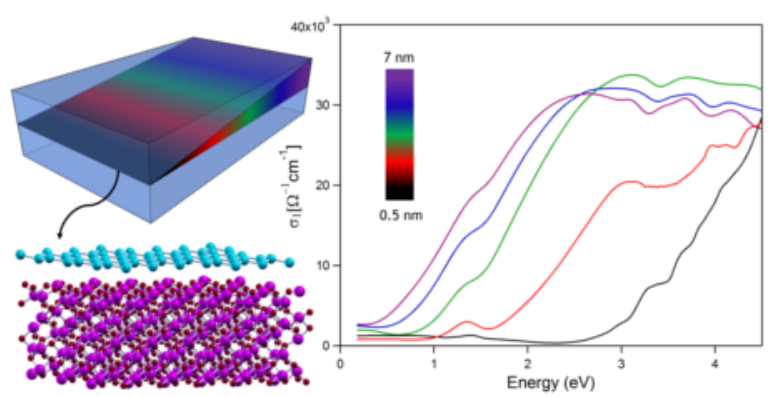

